\title{
Comparative sensitivity of fluorescent antibody staining of conjunctival scrapings and irradiated McCoy cell culture for the diagnosis of hyperendemic trachoma
}

\author{
S. DAROUGAR, ${ }^{1}$ R. M. WOODLAND,${ }^{1}$ B. R. JONES, ${ }^{1}$ \\ A. HOUSHMAND, ${ }^{2}$ AND H. A. FARAHMANDIAN ${ }^{3}$ \\ From the ${ }^{1}$ Department of Clinical Ophthalmology, Institute of Ophthalmology, Judd Street, \\ London WC1, the ${ }^{2}$ School of Public Health, University of Teheran, PO Box 1310, Teheran, Iran, \\ and the ${ }^{3}$ Department of Health and Welfare, Bandar Abbas, Iran
}

SUMMARY The sensitivity of an indirect fluorescent antibody (FA) staining technique for detecting chlamydial inclusions in scrapings from the whole conjunctiva (upper tarsus, upper fornix, and lower lid) was compared with the sensitivity of culture in irradiated McCoy cells for the diagnosis of hyperendemic trachoma. In a group of 211 patients with various grades of active trachoma from the Bandar Abbas area of Southern Ir an 42 patients were positive for chlamydiae by either method. There was little difference between the rates of positivity of FA staining of the scrapings from the whole conjunctiva ( 28 positives) and culture in irradiated McCoy cells ( 32 positives). In the patients included in this study chlamydial inclusions were detected in 15 eyes by examination of FA stained scrapings taken from the upper tarsal conjunctiva, whereas inclusions were detected in 40 eyes by the additional examination of scrapings taken from the upper fornix and lower lid $(P<0.001)$. The examination of FA stained scrapings taken from the whole conjunctiva and spread as a single but larger smear may provide a satisfactory alternative to cell culture methods for the diagnosis of trachoma, particularly for field studies when cell culture facilities are not available.

Laboratory tests which provide support for field studies of hyperendemic trachoma include the direct demonstration of chlamydial inclusions in conjunctival scrapings and cultural tests. The fluorescent antibody (FA) staining technique is more sensitive than the traditional method of Giemsa staining for the detection of chlamydial inclusions in conjunctival scrapings. ${ }^{1-4}$ Culture in irradiated McCoy cells is more sensitive than the direct demonstration of chlamydial inclusions in Giemsa stained smears for the diagnosis of hyperendemic trachoma as well as paratrachoma of sexually transmitted origin. ${ }^{4}$

The aims of the present study were to investigate the prevalence of chlamydial inclusions detected by FA staining in scrapings from the different areas of the conjunctiva and to compare the sensitivity of FA staining of scrapings from the upper tarsus as well as the whole conjunctiva with that of irra-

Correspondence to Dr S. Darougar, Institute of Ophthalmology, Judd Street, London WC1H 9QS. diated McCoy cell culture for the detection of Chlamydia trachomatis in cases of hyperendemic trachoma.

\section{Materials and methods}

SELECTION OF PATIENTS

Patients with different grades of active trachomatous inflammatory changes in their eyes ${ }^{5}$ were selected from 2 villages in the Bandar Abbas area of Southern Iran. These patients were part of a study on the natural history and transmission of hyperendemic trachoma in their villages.

\section{COLLECTION OF SPECIMENS}

Conjunctival swabbings were collected from the whole conjunctiva (i.e., the upper tarsus, upper fornix, and lower lid) of each eye as described by Darougar et al. ${ }^{6}$ Right and left paired specimens were pooled in a plastic capsule containing 2SP transport medium ${ }^{7}$ with additional $3 \%$ fetal bovine serum. All conjunctival swabbings were stored 
immediately in a portable liquid nitrogen refrigerator at $-180^{\circ} \mathrm{C}$ for transport to the Virus Laboratory, London, where they were subsequently stored in a refrigerator at $-70^{\circ} \mathrm{C}$ until inoculated into cell cultures.

After swabbing, conjunctival scrapings were collected separately from the upper tarsus, upper fornix, and lower lid of each eye with sterile aluminium scrapers. ${ }^{6}$ The material collected from each area was spread thinly and evenly within $6 \mathrm{~mm}$ diameter areas scratched on clean glass microscope slides. Scrapings were air dried and fixed in methyl alcohol for 5 minutes at room temperature. Transport to London (4 or 5 days) was at ambient temperature, after which slides were stored at $-20^{\circ} \mathrm{C}$ until stained.

\section{CELL CULTURE}

The simplified technique of culture in irradiated McCoy cells as described by Darougar et al. ${ }^{8}$ was used. Monolayers were fixed after 60-72 hours incubation, stained with Giemsa, and examined by dark-field illumination.

\section{FLUORESCENT ANTIBODY STAINING}

The conjunctival scrapings were stained by the indirect method of FA staining. ${ }^{9}$ The antiserum used, a hyperimmune rabbit serum against an LGV strain (LGV/2/USA/Cal-434/Bu), was produced at the Institute of Ophthalmology by multiple intravenous injections of agent over several months. The stained preparations were examined with a Zeiss standard fluorescence microscope fitted with an HBO 200 mercury vapour lamp and dark-field condenser. Exciter filter BG12 and barrier filters 50 and 44 were used. Bright green, sharply circumscribed inclusions within the cytoplasm of epithelial cells were identified as chlamydial inclusions. The total number of inclusions detected in each smear was recorded.

McNemar's test and paired sample $t$ test were used for comparison of the results.

\section{Results}

Of a total of 211 patients included in this study 42 patients $(20 \%)$ were positive by either FA or cell culture (Table 1). Of these 42 positive patients 28 $(67 \%)$ were positive by FA staining of scrapings from the whole conjunctiva, $12(29 \%)$ were positive by the examination of scrapings from the upper tarsus alone (the traditional method), and $32(76 \%)$ were positive by the irradiated McCoy cell culture method. The examination of FA stained scrapings from the upper tarsus alone was significantly less sensitive than cell culture $(P<0.001)$, but the
Table 1 Comparative sensitivity of FA staining of scrapings from the whole conjunctiva and culture in irradiated McCoy cells in 42 patients positive for chlamydiae by either method

\begin{tabular}{ll}
\hline & $\begin{array}{l}\text { Number of } \\
\text { patients }\end{array}$ \\
\hline Positive by cell culture & $32(76 \%)$ \\
Positive by FA & $28(67 \%)$ \\
Positive by FA and culture & $18(43 \%)$ \\
FA positive, culture negative & $10 *(24 \%)$ \\
FA negative, culture positive & $14(33 \%)$ \\
\hline
\end{tabular}

*The number of inclusions detected was fewer than 6 in 9 patients, whereas in the other patients it was 39 .

Table 2 Comparison of scrapings from different areas of the conjunctiva for the detection of chlamydial inclusions in 40 positive eyes

\begin{tabular}{lll}
\hline Source & $\begin{array}{l}\text { Number of } \\
\text { positive } \\
\text { scrapings }\end{array}$ & $\begin{array}{l}\text { Total } \\
\text { number of } \\
\text { inclusions }\end{array}$ \\
\hline Upper tarsus (UT) & $15(38 \%)$ & 60 \\
Upper fornix (UF) & $24(60 \%)$ & 81 \\
Lower lid (LL) & $28(70 \%)$ & 96 \\
Whole eye* (UT + UF + LL) & $40(100 \%)$ & 237
\end{tabular}

*The figures for the whole eye were obtained by combining the results for each individual area.

difference between the sensitivities of cell culture and FA staining of scrapings from the whole conjunctiva was not statistically significant $(P>0 \cdot 5)$.

In 28 patients who were positive by FA staining chlamydial inclusions were detected in conjunctival scrapings from 40 eyes. The number of positive smears from different areas of the conjunctiva and the numbers of inclusions detected in these 40 eyes are shown in Table 2. The lower lid showed the highest rate of $70 \%$ positivity ( 28 out of 40 ) with an average number of 2.4 inclusions per smear, whereas the upper tarsus was positive in only $38 \%$ (15 out of 40 ) with an average number of 1.5 inclusions. The difference between the rates of positivity of the upper tarsus and the lower lid is statistically significant $(P<0 \cdot 01)$. The number of inclusions seen in scrapings taken from the different areas of the conjunctiva were not significantly different from one another $(P>0 \cdot 1)$. In the case of only one eye was the scraping from the upper tarsus positive, while those from the upper fornix and the lower lid were negative. The examination of scrapings from the 3 different areas of the conjunctiva was significantly more sensitive than the examination of scrapings from any one area alone $(P<0.001)$. However, the addition of the results obtained from 
the upper tarsus scrapings did not significantly increase the sensitivity obtained by the examination of only the upper fornix and lower lid scrapings $(\mathrm{P}>0 \cdot 9)$.

\section{Discussion}

There was little difference between the sensitivities of cell culture and FA staining of scrapings from the whole conjunctiva for the diagnosis of trachoma. In this study the conjunctivae were swabbed for culture before scraping, which may have reduced the rate of detection of inclusions in the scrapings, especially where few inclusions were present. However, studies in London have shown that the order in which conjunctival scrapings and swabbings are collected has little effect on the rate of positivity of either type of specimen. ${ }^{10}$

For FA staining the World Health Organisation ${ }^{11}$ recommends fixation in acetone, transport on wet ice, and storage at $-20^{\circ} \mathrm{C}$. In this investigation scrapings were fixed in methyl alcohol and transported to London at ambient temperature, a method which is not considered optimum for subsequent FA staining. It is therefore possible that some inclusions present in the scrapings were not detected.

The World Health Organisation ${ }^{11}$ recommends that conjunctival scrapings are taken from only the upper tarsus. The results of this study indicate that scrapings from this area are the least sensitive for the detection of inclusions. Scrapings from the upper fornix and the lower lid were almost twice as sensitive as scrapings from the upper tarsus for the diagnosis of trachoma. However, for maximum sensitivity, scrapings should be taken from all areas of the conjunctiva. In this study only 1 eye had inclusions in the upper tarsus alone, and it may therefore be possible to exclude the upper tarsus scrapings without significantly reducing the sensitivity of the test.

The time required for the examination of a single FA stained scraping is approximately the same as for the examination of a McCoy cell monolayer. The examination of scrapings from different areas of the conjunctiva is therefore more time-consuming than the cell culture method. However, if material taken from different areas were spread on a single but larger smear, there should be little difference between the times taken for the 2 methods. The time needed to prepare the specimens for examination is similar for either method, but conjunctival scrapings can be stained by the FA technique immediately and results can be obtained within 2 to 3 hours of fixation.

While cell culture is the method of choice for the diagnosis of chlamydial infections, an established laboratory with experienced technicians and adequate facilities for the transport of specimens while frozen is required for this method. However, FA staining of scrapings from the whole conjunctiva offers a satisfactory alternative for which the main requirements are antisera and a fluorescence microscope, and may therefore be better suited for studies in the field where facilities for cultural tests are not generally available. The use of both FA and cell culture procedures is to be recommended if maximum sensitivity is required.

The authors are grateful to the Dean of the School of Public Health, Teheran, Iran, and the Director of the Department of Health and Welfare, Bandar Abbas, Iran, for their support of this project.

We are indebted to $\mathrm{Mr} \mathrm{H}$. Shah Mohammadi for his assistance in the collection of clinical specimens.

This project was partially supported by grants from the Dulverton Trust, the Wellcome Foundation, the Order of St John, and an anonymous donor.

\section{References}

${ }^{1}$ Nichols RL, Bobb AA, Haddad NA, McComb DE. Immunofluorescent studies of the microbiologic epidemiology of trachoma in Saudi Arabia. Am J Ophthalmol $1967 ; 63: 1372-424$.

${ }^{2}$ Hanna L. An evaluation of the fluorescent antibody technique for the diagnosis of trachoma and inclusion conjunctivitis. Rev Int Trach 1968; 45: 345-59.

${ }^{3}$ Sowa J, Collier LH, Sowa S. A comparison of the iodine and fluorescent antibody methods for staining trachoma inclusions in the conjunctiva. J Hyg (Camb) 1971; 69: 693-708.

${ }^{4}$ Darougar S, Dwyer RStC, Treharne JD, Harper IA, Garland JA, Jones BR. A comparison of laboratory methods of diagnosis of chlamydial infection. In: Nicholas RL, ed. Trachoma and Related Disorders Caused by Chlamydial Agents. Amsterdam: Excerpta Medica, 1971; 445-60.

${ }^{5}$ Dawson CR, Jones BR, Darougar S. Blinding and nonblinding trachoma: the assessment of intensity of upper tarsal inflammatory disease and blinding lesions. Bull WHO 1975; 52: 279-82.

${ }^{8}$ Darougar S, Treharne JD, Dwyer RStC, Kinnison JR, Jones BR. Isolation of TRIC agent (Chlamydia) in irradiated McCoy cell culture from endemic trachoma in field studies in Iran. Br J Ophthalmol 1971; 55: 591-9.

${ }^{7}$ Gordon FB, Harper IA, Quan AL, Treharne JD, Dwyer RStC, Garland JA. Detection of Chlamydia (Bedsonia) in certain infections of man. I. Laboratory procedures: comparison of yolk sac and cell culture for detection and isolation. J Infect Dis 1969; 120: 451-62.

${ }^{8}$ Darougar S, Kinnison JR, Jones BR. Simplified irradiated McCoy cell culture for isolation of Chlamydiae. In: Nichols RL, ed. Trachoma and Related Disorders Caused by Chlamydial Agents. Amsterdam: Excerpta Medica, $1971 ; 63-70$.

${ }^{9}$ Schachter J, Dawson CR, Balas S, Jones P. Evaluation of laboratory methods for detecting acute TRIC agent infections. Am J Ophthalmol 1970; 70: 375-80.

${ }^{10}$ Darougar S, Jones BR. Conjunctival swabbing for the isolation of TRIC agent (Chlamydia). Br J Ophthalmol 1971; 55: 585-90.

"World Health Organisation. Guide to the Laboratory Diagnosis of Trachoma. Geneva, 1975. 